\title{
BH masses in NLS1: the role of the broad-line region geometry
}

\section{R. Decarli*}

Max-Planck Institut für Astronomie - Heidelberg

E-mail: decarli@mpia.de

\section{Dotti}

Università degli Studi di Milano-Bicocca

E-mail: massimo.dotti@mib.infn.it

\section{F. Haardt}

Università degli Studi dell'Insubria - Como

E-mail: francesco.haardteuninsubria.it

\section{S. Zibetti}

DARK Cosmology center - Copenhagen

E-mail: zibetti@dark-cosmology.dk

\begin{abstract}
Narrow Line Seyfert 1 galaxies (NLS1) are generally believed to host “under-massive" black holes with respect to the predictions from the host galaxy - black hole mass scale relations. Black hole masses in NLS1 are estimated from the continuum luminosity and the width of broad emission lines. Here we show that the "mass deficit" can be canceled out if we assume that the broad line region (BLR) in type-1 AGN has a flat geometry, which is seen face-on in NLS1. The detection of relativistic jets aligned along the line of sight in a number of NLS1 supports this picture. Moreover, a flat geometry of the BLR is also suggested by a general trend of the mass deficit as a function of the line width observed in other type-1 AGN, from quasars to BL Lac objects, and is consistent with a simple extension of the Unified Model of AGN to the BLR geometry.
\end{abstract}

Narrow-Line Seyfert 1 Galaxies and their place in the Universe - NLS1,

April 04-06, 2011

Milan Italy

\footnotetext{
* Speaker.
} 


\section{Introduction}

Narrow-line Seyfert 1 galaxies (NLS1) are a peculiar class of type-1 Active Galactic Nuclei $(\mathrm{AGN})$ characterized by a modest width of permitted broad lines $\left(\mathrm{FWHM}=500-2000 \mathrm{~km} \mathrm{~s}^{-1}\right)$, weak [O III] and strong Fe II emission lines [1, 2], strong variability, and a soft X-ray excess [3, 4]. They represent about $15 \%$ of the whole population of Seyfert 1 galaxies [5], from optical spectroscopy classification.

In the context of the joint evolution of massive black holes (BHs) and their host galaxies, the most important feature of NLS1 is that they have, on average, lower $M_{\mathrm{BH}}$ than expected from $M_{\mathrm{BH}^{-}}$ host galaxy relations [6], while $M_{\mathrm{BH}}$ in normal broad line Seyfert galaxies (hereafter, BLS1) are in fairly good agreement to the same relation. On the other hand, AGN luminosities in NLS1 are comparable to those of BLS1. Therefore, the " $M_{\mathrm{BH}}$ deficit" directly yields high Eddington ratios $L / L_{\mathrm{Edd}}$ in NLS1 [7]. Relatively modest BH masses are found from variability studies of some NLS1 $[8,9,10]$ and are sometimes associated with peculiar properties of the host galaxy, e.g., pseudo-bulges [11] or enhanced star formation [12]. In a BH-galaxy co-evolution scenario, NLS1 could be still on their way to reach the $M_{\mathrm{BH}}-\sigma_{*}$ relation, i.e., their (comparatively) small BHs are highly accreting in already formed bulges, thus moving towards the BLS1 relation.

However, $M_{\mathrm{BH}}$ in type-1 AGN are usually estimated through the so-called virial paradigm, according to which the clouds in the broad line region (BLR) orbit around the singularity following purely-gravitational dynamics. Under this assumption,

$$
M_{\mathrm{BH}}=G^{-1} R_{\mathrm{BLR}} v_{B L R}^{2},
$$

where $G$ is the gravitational constant, and $R_{\mathrm{BLR}}, v_{B L R}$ are the broad line region characteristic size and the velocity of broad-line emitting clouds. $R_{\mathrm{BLR}}$ is found by means of the reverberation mapping technique [16], or through the $R_{\mathrm{BLR}}$-luminosity relations $[17,18,19]$. The width of the broad emission lines, parametrized e.g. by the full width at half maximum (FWHM), is used as a proxy for $v_{\mathrm{BLR}}$, assuming a deprojection factor $f$ :

$$
v_{\mathrm{BLR}}=f \cdot \mathrm{FWHM}
$$

The value of $f$ is generally unknown and depends on some assumptions about the geometry of the BLR. In the simple, isotropic scenario $f=\sqrt{3 / 4}$ [13]. If the BLR is rotationally supported (see, e.g., $[14,15])$, an orientation dependence is introduced. If $\vartheta$ is the angle between the rotation axis and the line of sight, the value of $f$ for a disk would be:

$$
f=\frac{1}{2}\left[\left(\frac{H}{R}\right)^{2}+\sin ^{2} \vartheta\right]^{-1 / 2}
$$

where $H$ and $R$ are the vertical and radial scale lengths of the disk. For infinitely thin disk, $f$ ranges between 0.5 and infinity as $\vartheta$ changes from 90 (edge-on) to 0 (pole-on) degrees. Assuming disks with finite thickness would set an upper limit to $f, f_{\max }=R /(2 H)$.

In order to measure $f$, two independent estimates of $M_{\mathrm{BH}}$ are necessary, only one of which is based on equations 1.1-1.3. The most common approach is to assume that the BH follows the $M_{\mathrm{BH}}$-host galaxy relations, therefore the host properties (e.g., $\sigma_{*}$ or the bulge luminosity) can be 

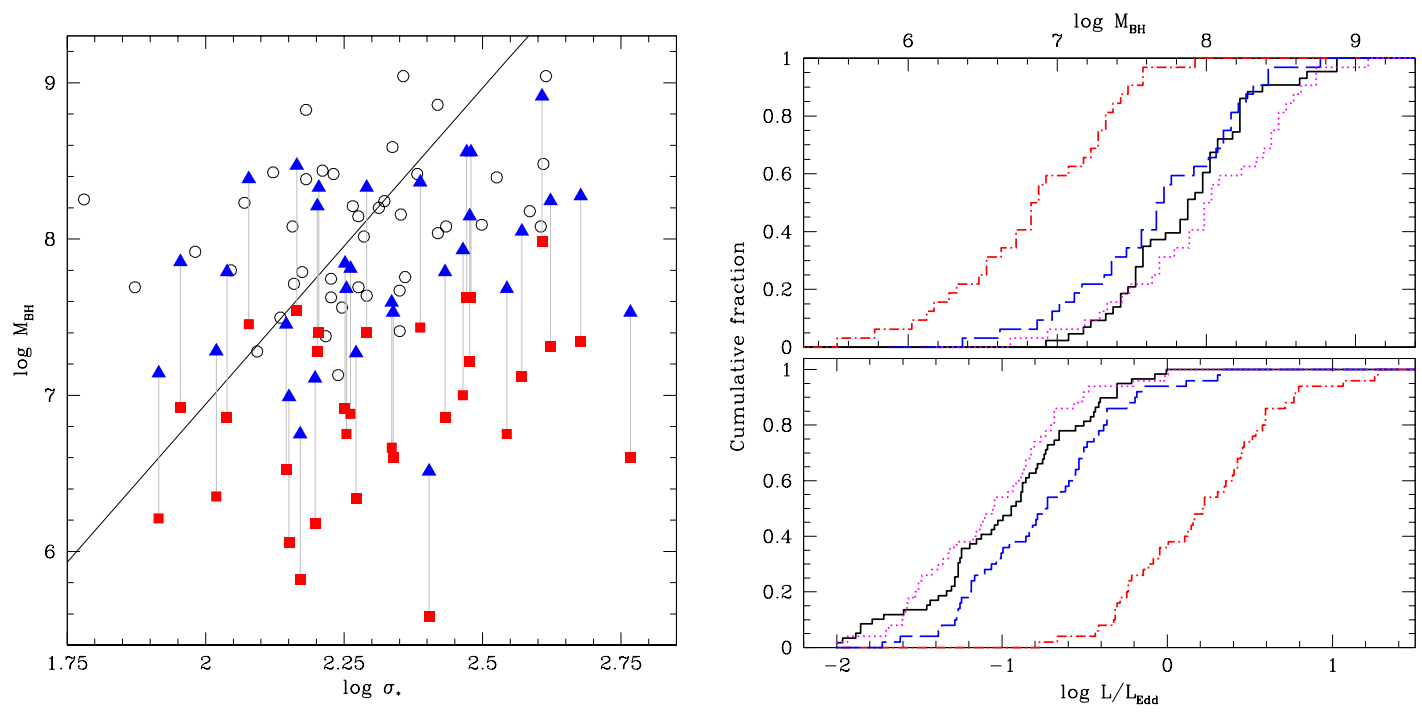

Figure 1: Left panel: The distribution of BLS1 (empty, black circles) and NLS1 (squares and triangles) in the $\left(\sigma_{*}, M_{\mathrm{BH}}\right)$ plane. Data are taken from [26]. Red squares refer to the isotropic case, while blue triangles refer to the disk-like BLR case with $H / R=0.1$. After correcting for the BLR geometry, the two AGN classes populate the same region of the plot. Right panel: the cumulative distribution of $M_{\mathrm{BH}}$ (top) and $L / L_{\mathrm{Edd}}$ (bottom) of BLS1 (black, solid lines) and NLS1. The red, dot-dashed line is obtained assuming an isotropic BLR, with $f=\sqrt{3 / 4}$. The blue, dashed line and the magenta, dotted lines are obtained assuming a disk-like BLR with $H / R=0.1$ and 0 respectively. The match with the $M_{\mathrm{BH}}$ and $L / L_{\mathrm{Edd}}$ distributions of normal BLS1 is remarkable. Thin disks with $0<H / R<0.1$ best fit the distributions. Figure adapted from [27].

used as a proxy of $M_{\mathrm{BH}}[20,21,22,23]$ (see however the caveats discussed in by Mathur [7] and [24]). All these studies found average values of $f$ exceeding unity, in constrast with the simple expectations from the isotropic model. In this picture, the observed small FWHM of NLS1 broad lines may be ascribed to a small viewing angle with respect to the disc axis, hence no evolutionary difference is required.

Here we investigate this latter scenario from three different point of views: the demography of NLS1 with respect to BLS1 (section 2); the geometric deprojection factor $f$ as derived in objects with small inclination angles $\vartheta$ (section 3); and the comparison with the general quasar properties in terms of virial black hole masses versus host galaxy properties (section 4). Conclusions are drawn in section 5 .

\section{Breaking a cosmic conspiracy?}

The key point of the orientation scenario is the assumption that the BLR has a flat geometry, and that the relatively narrow width of permitted lines in NLS1 galaxies is due to small inclination angles (pole-on views) rather than intrinsically narrow lines. This implies an underestimate of the virial $M_{\mathrm{BH}}$, and consequently an overestimate of $L / L_{\mathrm{Edd}}$. Under this hypothesis, we can estimate the average value of $f$ for NLS1 and BLS1 simply requiring that the solid angle of pole-on systems 
and the one of non pole-on systems match the relative fractions of NLS1 and BLS1. According to the Unified Model of AGN, type-1 systems have inclination angles ranging between 0 and $\vartheta_{\text {max }}$ $=40-60$ degrees [25]. We can define a $\vartheta_{\text {cut }}$ so that objects with $\vartheta<\vartheta_{\text {cut }}$ are labeled as NLS1, while objects with $\vartheta_{\text {cut }}<\vartheta<\vartheta_{\max }$ are considered BLS1. NLS1 constitute $15 \%$ of the BLS1 population, which yields $\vartheta_{\text {cut }} \approx 15^{\circ}$, the exact value depending on $H / R$. The corresponding average deprojection factors are $\langle f\rangle_{\mathrm{NLS} 1} \approx 3$ and $\langle f\rangle_{\mathrm{BLS} 1} \approx 1$ (see [27] for details).

Given these values, the position of BLS1 in the $\left(\sigma_{*}, M_{\mathrm{BH}}\right)$ plane is practically unchanged with respect to the isotropic case assumed, e.g., in [26]. On the other hand, BH masses in NLS1 galaxies are shifted $\approx 1$ dex upwards, thus matching the expectations from the $M_{\mathrm{BH}^{-}} \sigma_{*}$ relation. Similarly, the Eddington ratios are lowered by $\approx 1 \mathrm{dex}$, matching the distribution observed in normal BLS1.

It is remarkable that with the only assumption that BLR is flat in all type-1 Seyfert galaxies, and that they all follow the BH-host galaxy relations, we can break the "cosmic conspiracy" yielding relatively low $M_{\mathrm{BH}}$ and similarly high $L / L_{\mathrm{Edd}}$ in AGN having the same range of AGN luminosity and host galaxy properties (in this case, $\sigma_{*}$ ). Once the orientation effect are taken into account, the distributions of $M_{\mathrm{BH}}$ and $L / L_{\mathrm{Edd}}$ in NLS1 and BLS1 are in good agreement as well (see Figure 1).

\section{The realm of small inclination angles}

In order to test the orientation scenario, one would ideally have an independent way to measure the inclination. We therefore focus now on a class of type-1 AGN in which inclination angles are known to be small. Blazars, and BL Lac objects among them, are characterized by strong continuum luminosity in the optical and radio wavelengths, associated to the synchrotron emission from a relativistic jet almost aligned with the line of sight, i.e., $\vartheta \lesssim 10^{\circ}[28,25]$. Therefore, we expect BL Lac objects to show substantially higher $f$ values than the typical quasars.

We select a sample of quasars and blazars requiring that the host galaxy has been resolved and it's elliptical (so that $L_{\text {bulge }} \approx L_{\text {host }}$ ), and that broad $\mathrm{Mg}$ II or $\mathrm{H} \alpha$ lines have been observed in their optical spectra. Quasars are taken from [29, 30], while blazars are collected mainly from $[31,32,33]$. The full list of sources and references for the data is given in [34]. The final sample consists of 18 blazars (including 8 BL Lac objects) and 39 quasars.

Virial BH masses are derived for each object using the broad line width (FWHM) and luminosity $\left(L_{\text {line }}\right)$ used as a proxy of the accretion disk continuum luminosity ${ }^{1}$. The host galaxy stellar mass $\left(M_{\text {host }}\right)$ is derived from the rest-frame $R$-band luminosity of the host galaxy, after applying $k$-correction and assuming the mass-to-light ratio of a single stellar population formed at high redshift and passively evolving (see [30, 34]). Finally, we adopt the $M_{\mathrm{BH}} / M_{\text {host }}$ ratio and its redshift dependence as found in [30] in order to compute $f$.

Blazars, and in particular BL Lac objects, show important (up to 2 dex!) deviations with respect to the local $M_{\mathrm{BH}} / M_{\text {host }}$ relation, if the geometrical factor is not taken into account (see Figure 2, left). Most importantly, the geometrical factor $f$ shows a clear dependence on the line width (see Figure 2, right), so that objects with modest line width have high ( $>3$ ) values of $f$.

\footnotetext{
${ }^{1} \mathrm{~A}$ fraction of the photoionizing radiation illuminating the BLR in blazars could in principle come from the jet, rather than from the accretion disk. However, line luminosities are found to be independent of the jet-associated continuum [35].
} 

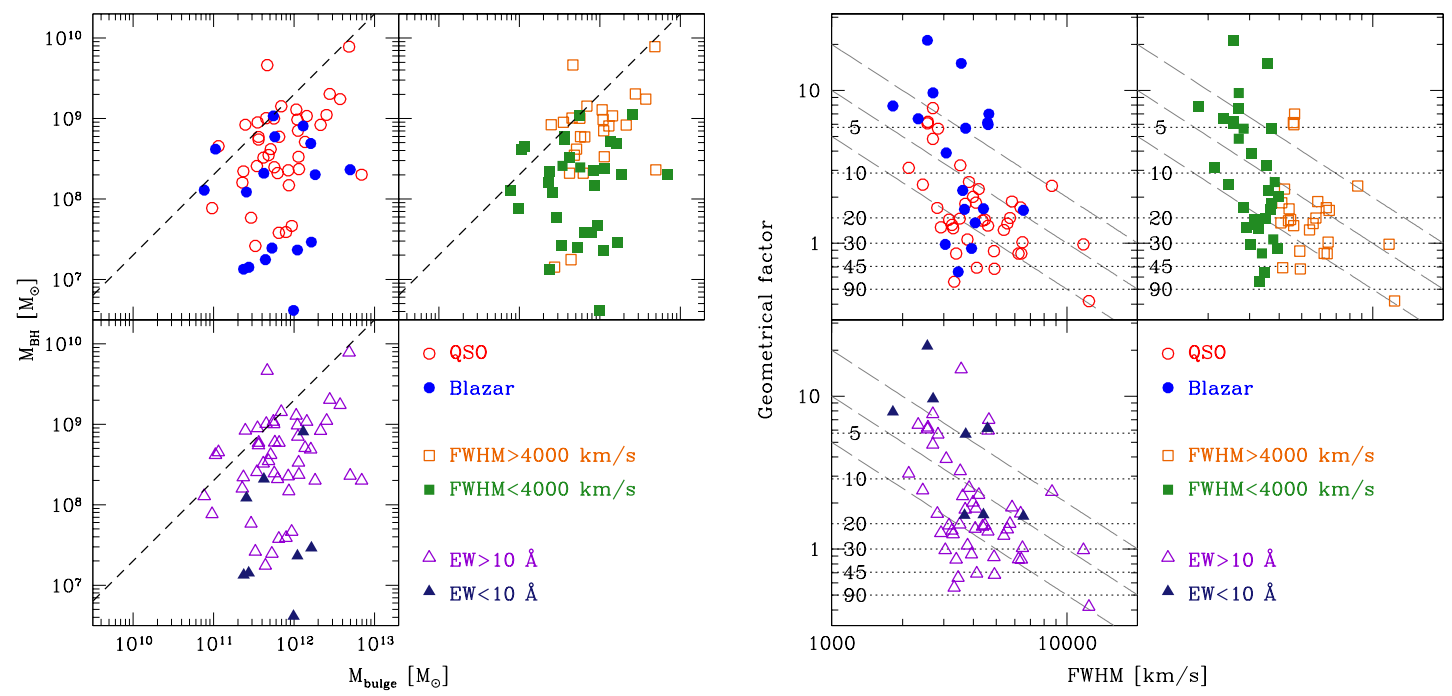

Figure 2: Left panel: the $\left(M_{\text {host }}, M_{\mathrm{BH}}\right)$ plane in quasars and blazars in our sample. Virial $M_{\mathrm{BH}}$ with $f=1$ are plotted along the $y$-axis. Blazars, and especially BL Lac objects (highlighted by the $\mathrm{EW}<10 \AA$ cut) systematically deviate from the local $M_{\mathrm{BH}}=0.0015 M_{\text {host }}$ relation (dashed line). Right panel: the geometrical factor $f$ as a function of the line width. A clear trend is apparent, with $f$ decreasing as the line width increase. In particular, we note that objects with relatively narrow lines $\left(<4000 \mathrm{~km} \mathrm{~s}^{-1}\right)$ have systematically higher $f$ values, consistently with the expectations for a thin disk-like BLR as seen from various inclination angles (5, 10, 20, 30, 45 and 90 degrees, horizontal dotted lines in the plot; see equation 1.3). BL Lac objects, known to have small $\vartheta$, populate the top-left corner of this plot, with relatively narrow lines and high $f$, consistently with the finding for NLS1 described in section 2. Figure adapted from [34].

It is remarkable that blazars, and specifically BL Lac objects, populate the top-left corner of the (FWHM, $f$ ) plane, with relatively narrow lines and high deprojection factors, consistently with the disk-like picture of the BLR. This trend is in bitter disagreement with the isotropic model of the BLR, according to which the geometrical factor is constant and smaller than 1. It is interesting to note that some objects have very high $f$ values, thus supporting the idea of a geometrically thin $(H / R<0.1)$ disk.

\section{Another look at the $M_{\mathrm{BH}}-M_{\text {host }}$ relation in quasars}

The study presented in section 3 is strongly limited by the modest size of quasar and blazar samples with resolved host galaxy available in the literature. This is because the extended component associated to the galaxy is outshone by the bright nuclei, and optimal angular resolutions are required to probe the host galaxy. However, it is possible to derive average properties of quasar host galaxies from stacking several hundred images of quasars observed with non-optimal seeing conditions, e.g., from the Sloan Digital Sky Survey. The power of this approach is shown in Figure 3, left, where we show the "net" images of stacked quasars at $0.3<z<0.4$ binned as a function of their virial BH mass. Net images are obtained by subtracting from the quasar image the image of a bright star in the field, scaled to match the central surface brightness of the target. Since host galax- 

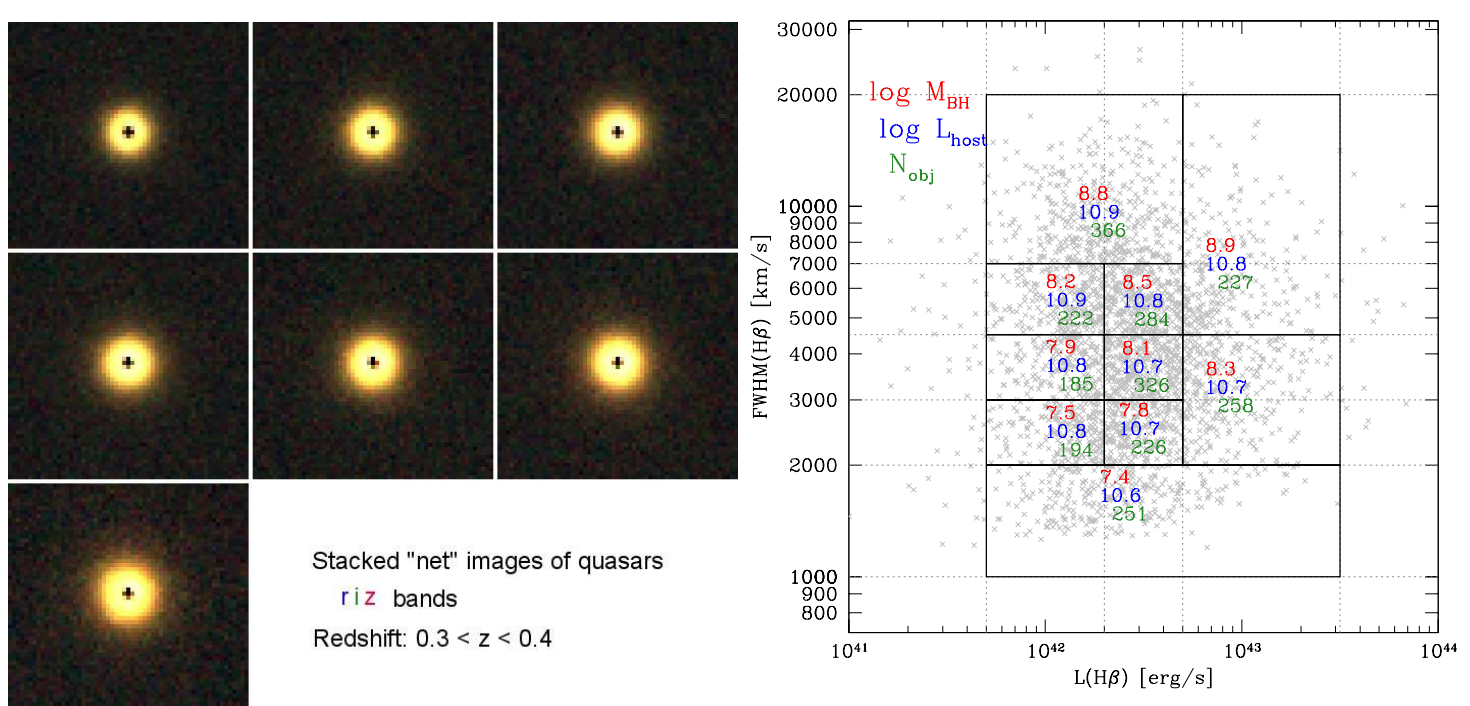

Figure 3: Left panel: stacked "net" images of quasars at $0.3<z<0.4$ in various $M_{\mathrm{BH}}$ bins. The RGB images are created as a RGC composite of the SDSS zir stacks. Net images are obtained by subtracting from the quasar image the image of a bright field star, scaled to match the cental brightness of the target. The extended emission of the host galaxies is clearly detected after the stacking analysis. Right panel: the ( $L_{\text {line }}$, FWHM) plane for SDSS quasars at $0.3<z<0.4$ (grey crosses). Data are taken from [37]. We rebinned the sample in blocks of comparable FWHM and $L_{\text {line }}$ (thick black lines), and computed the average $M_{\mathrm{BH}}$ in each bin (values in red). As expected, $M_{\mathrm{BH}}$ increases towards to top-right corner of the plot. We then performed our stacking analysis on each bin. The resulting host galaxy luminosity is shown in blue. While the FWHM increases by a factor $\sim 5$ from bottom to top ( $M_{\mathrm{BH}}$ increases by a factor $\left.\sim 20\right)$, only a small increase in terms of $L_{\text {host }}$ is reported (a factor $\sim 2$ ), suggesting that the line width, taken without any correction for the $f$-FWHM dependence, is not a good tracer of $M_{\mathrm{BH}}$.

ies are rarely resolved in typical SDSS data, individual net images are noise-dominated. However, once we stack few hundred of sources, the extended light from the host galaxy is apparent.

We performed our stacking analysis on SDSS quasars ranging between $z=0.3$ and $z=1.3$ (in order to have significant host galaxy emission in the SDSS filters), and binning as a function of spectral properties of the quasars, e.g., $M_{\mathrm{BH}}$, FWHM or luminosity of some broad line, etc. Then we modeled the stacked image of the quasars using a Sersic profile for the host galaxy and the PSF model derived from the stacked image of field stars. The resulting magnitudes in 4 bands (SDSS griz) are then compared to get color information and to give constraints on the stellar population of the host galaxies. This allows us to probe the dependence of the average $L_{\text {host }}$ and $M_{\text {host }}$ in each bin as a function of the quasar properties and the redshift. Details will be presented in [36].

In Figure 3, right we plot the $0.3<z<0.4$ quasars from [37] in the ( $L_{\text {line }}$, FWHM) plane. We binned the plane in terms of line width and luminosity, and computed the average virial $M_{\mathrm{BH}}$ in each bin. Then, we perfomed our stacking analysis, and evaluated the average host galaxy luminosity. Surprisingly, while the FWHM increases by a factor $\sim 5$ from the bottom to the top of the plot, and the average $M_{\mathrm{BH}}$ increases by a factor $\sim 20$, the host galaxy luminosity changes only by a factor $\sim 2$ from the bottom to the top of the diagram. This result is in disagreement with the observed 
$M_{\mathrm{BH}}-M_{\text {host }}$ relation. If, as shown before, the geometrical factor shows a dependence on the FWHM, then the FWHM could have only a limited power in predicting the actual $v_{\mathrm{BLR}}$. This could explain the lack of correlation between $M_{\mathrm{BH}}$ and host luminosities from the stacking analysis, as bins are defined on virial $M_{\mathrm{BH}}$ estimates.

\section{Conclusions}

We showed that the relatively small $M_{\mathrm{BH}}$ and high $L / L_{\mathrm{Edd}}$ values observed in NLS1 may be fully attributed the orientation of the BLR with respect to the line of sight, once a flat BLR geometry is assumed. This argument can simultaneously break the "cosmic conspiracy" in NLS1, explain the mismatch between virial $M_{\mathrm{BH}}$ and $M_{\mathrm{BH}}$ obtained from the host galaxy properties in blazars, and accounts for the lack of any significant correlation between virial $M_{\mathrm{BH}}$ and host galaxy luminosities in quasars.

\section{References}

[1] Osterbrock D.E., Pogge R.W., 1985, ApJ, 297, 166

[2] R. W. Pogge: A quarter century of Narrow-Line Sefert 1s. In: Proceedings of the Workshop Narrow-Line Seyfert 1 Galaxies and Their Place in the Universe, eds L. Foschini, M. Colpi, L. Gallo, D. Grupe, S. Komossa, K. Leighly, \& S. Mathur. Proceedings of Science (NLS1) 002 (2011).

[3] Boller T., Tanaka Y., Fabian A., Brandt W.N., Gallo L., Anabuki N., Haba Y., Waughan S., 2003, MNRAS, 343, L89

[4] Grupe D., Beuermann K., Mannheim K. Thomas H.C., 1999, A\&A, 350, 805

[5] Williams R.J., Pogge R.W., Mathur S., 2002, AJ, 124, 3042

[6] Mathur S., et al. 2001, New Astronomy, 6, 321

[7] S. Mathur: Host galaxies of NLS1s. In: Proceedings of the Workshop Narrow-Line Seyfert 1 Galaxies and Their Place in the Universe, eds L. Foschini, M. Colpi, L. Gallo, D. Grupe, S. Komossa, K. Leighly, \& S. Mathur. Proceedings of Science (NLS1) 002 (2011).

[8] Green A.R., McHardy I.M., Lehto H.J., 1993, MNRAS, 265, 664

[9] Hayashida K., 2000, NewAR, 44, 419

[10] McHardy I.M., Koerding E., Knigge C., Uttley P., Fender R.P., 2006, Nature, 444, 730

[11] R. Davies: Cosmic Evolution of NLS1 and the Growth of their Black Holes. In: Proceedings of the Workshop Narrow-Line Seyfert 1 Galaxies and Their Place in the Universe, eds L. Foschini, M. Colpi, L. Gallo, D. Grupe, S. Komossa, K. Leighly, \& S. Mathur. Proceedings of Science (NLS1) 002 (2011).

[12] E. Sani: Enhanced star formation in Narrow-Line Seyfert 1 galaxies. In: Proceedings of the Workshop Narrow-Line Seyfert 1 Galaxies and Their Place in the Universe, eds L. Foschini, M. Colpi, L. Gallo, D. Grupe, S. Komossa, K. Leighly, \& S. Mathur. Proceedings of Science (NLS1) 002 (2011).

[13] H. Netzer, 1990, in Blandford R.D., Netzer H., Woltjer L., eds, Active Galactic Nuclei, Springer, Berlin , p. 137

[14] Wills B.J., Browne I.W.A., 1986, ApJ, 302, 56

[15] Bian W. \& Zhao Y., 2004, MNRAS, 352, 823 
[16] Blandford R.D., McKee C.F., 1982, ApJ, 255, 419

[17] Kaspi S., Smith P.S., Netzer H., Maoz D., Jannuzi B.T., Giveon U., 2000, ApJ, 533, 631

[18] Kaspi S., Maoz D., Netzer H., Peterson B.M., Vestergaard M., Jannuzi B.T., 2005, ApJ, 629, 61

[19] Kaspi S., Brandt W.N., Maoz D., Netzer H., Schneider D.P., Shemmer O., 2007, ApJ, 659, 997

[20] Onken C.A., Ferrarese L., Merritt D., Peterson B.M., Pogge R.W., Vestergaard M., Wandel A., 2004, ApJ, 615, 645

[21] Labita M., Treves A., Falomo R., Uslenghi M., 2006, MNRAS, 373, 551

[22] Decarli R., Labita M., Treves A., Falomo R., 2008a, MNRAS, 387, 1237

[23] Graham A.W., Onken C.A., Athanassoula E., Combes F., 2011, MNRAS, 412, 2211

[24] Batcheldor D., 2010, ApJ, 711L, 108

[25] Urry C.M., Padovani P., 1995, PASP, 107, 803

[26] Grupe D., Mathur S., 2004, ApJ, 606L, 41

[27] Decarli R., Dotti M., Fontana M., Haardt F., 2008b, MNRAS Letters, 386, 15

[28] Ghisellini G., Padovani P., Celotti A., Maraschi L., 1993, ApJ, 407, 65

[29] Decarli R., Falomo R., Treves A., Kotilainen J.K., Labita M., Scarpa R., 2010a, MNRAS, 402, 2441

[30] Decarli R., Falomo R., Treves A., Labita M., Kotilainen J.K., Scarpa R., 2010b, MNRAS, 402, 2453

[31] Scarpa R., et al., 2000, ApJ, 532, 740

[32] Kotilainen J.K., Falomo R., \& Scarpa R., 1998, A\&A, 332, 503

[33] Nilsson K., Pursimo T., Heidt J., Takalo L.O., SillanpäÄ., Brinkmann W., 2003, A\&A, 400, 95

[34] Decarli R., Dotti M., Treves A., 2011, MNRAS, 413, 39

[35] Corbett E.A., Robinson A., Axon D.J., Hough J.H., 2000, MNRAS, 311, 485

[36] Decarli R., Zibetti S., 2011, in preparation

[37] Shen Y., Richards G.T., Strauss M.A., Hall P.B., Schneider D.P., Snedden S., Bizyaev D., Brewington H., et al., 2010, arXiv:1006.5178 